\title{
COMMUNICATION
}

\section{Ulcération persistante du plancher buccal : un cas de sialométaplasie nécrosante de la glande sublinguale?}

\author{
Alande $\mathbf{C}^{1}$, Fénelon $\mathbf{M}^{1}$, Catros S2, Fricain JC ${ }^{3}$ \\ 1. Service d'Odontologie et de santé buccale, Hôpital Pellegrin (interne DESCO) - Université Victor Segalen - Bordeaux II \\ 2. Médecine bucco-dentaire CHU Bordeaux - Université Victor Segalen - Bordeaux II \\ 3. Bioingénierie tissulaire (BIOTIS) \{ Inserm : U1026, Université Victor Segalen - Bordeaux ||
}

\section{Introduction}

La sialométaplasie nécrosante (SMN) est une affection bénigne, rare, à composante inflammatoire, touchant les glandes salivaires (Abrams AM et al. Cancer 1973;32:130-5). Cette pathologie affecte essentiellement les glandes salivaires accessoires du palais. La pathogénie n'est pas encore définitivement admise, mais la survenue d'un évènement ischémique précédent l'apparition de la lésion est létiologie la plus souvent suggérée. Cliniquement, la SMN est caractérisée par une ulcération cratériforme, aux bords surélevés, entourée d'un halo érythémateux, le plus souvent unilatérale, avec une taille variant de 1 à $3 \mathrm{~cm}$. L'évolution se fait spontanément vers la guérison dans un délai de 3 à 5 semaines. Histologiquement, la SMN est caractérisée par une nécrose des lobules des glandes salivaires accessoires, un infiltrat inflammatoire polymorphe, une métaplasie épidermoïde des canaux et acini adjacents, une absence d'atypie cellulaire, et une hyperplasie de l'épithélium adjacent à l'ulcération. L'architecture des glandes est conservée. Très peu de cas intéressant les glandes principales ont été décrit dans la littérature (AubiesTrouilh S. et al. Med Buccale Chir Buccale 2012;18:347-359). Un cas d'ulcération unilatérale du plancher buccal évocatrice d'une sialométaplasie nécrosante est présenté.

\section{Observation}

En octobre 2015, un patient de 29 ans a consulté pour une ulcération profonde du plancher buccal droit, douloureuse, ayant débutée en juillet 2015 et s'accompagnant d'une anesthésie de la pointe de la langue. Fin août 2015, une biopsie réalisée par un confrère avait conclu à une ulcération non spécifique de type bourgeon charnu. Après la consultation, un TPHA-VDRL et une sérologie HIV 1 et 2 ont été prescrits, ainsi qu'un hémogramme avec électrophorèse des protéines. L'ensemble du bilan biologique était normal. Une seconde biopsie a alors été réalisée, aboutissant aux mêmes conclusions que la première. Par élimination, un diagnostic de SMN intéressant la glande sublinguale a été porté. Devant l'absence de guérison spontanée, des traitements par injection de Kénacort 80mg puis par laser $\mathrm{CO} 2$ ont été instaurés, sans succès. Le patient décrivait cependant une amélioration des symptômes douloureux. En mars 2016, une reconstruction chirurgicale a été envisagée, mais du fait de l'amélioration des signes cliniques (amorce de cicatrisation), l'intervention n'a pas été réalisée. En juin 2016, on notait une étendue moins importante de la lésion, un aspect inflammatoire minoré et une reprise de la sensibilité de la pointe de la langue. Au mois de novembre 2016, malgré une amélioration, la cicatrisation totale de la lésion n'a pu être objectivée.

\section{Discussion}

Ce cas d'ulcération profonde non aphtoide était cliniquement évocateur d'une néoplasie maligne qui a éé infirmé par I'histologie. Les autres causes d'ulcération non aphtoide ont été éliminés sur des arguments cliniques ou biologiques : ulcération traumatique, infectieuse (syphillis, tuberculose, CMV, herpes), autoimmune (pemphigoïde, lichen plan), nécrolyse épidermique toxique. Le seul diagnostic compatible avec l'histoire clinique était une sialométaplasie nécrosante de par l'aspect de l'ulcération et la localisation unilatérale. L'examen anatomopathologique n'a pas permis de confirmer ce diagnostic mais les biopsies ont été réalisées à distance du début de l'ulcération ce qui pourrait expliquer l'aspect de bourgeon charnu.

cedric.alande@laposte.net

(C) The authors, published by EDP Sciences. This is an Open Access article distributed under the terms of the Creative Commons Attribution License 4.0 (http://creativecommons.org/licenses/by/4.0/). 